\title{
The Challenges of the Health Care Providers in Refugee Settings: A Systematic Review
}

\author{
Nurtaç Kavukcu, MA, MSc-candidate; ${ }^{1}$ Kerim Hakan Altıntaş, MD, EMDM ${ }^{2} \odot$
}

\section{Department of Public Health, Institute of Health Sciences, Hacettepe University, Ankara, Turkey \\ 2. Faculty of Medicine, Department of Public Health, Hacettepe University, Ankara, Turkey \\ Correspondence: \\ Kerim Hakan Altintas, MD, EMDM Department of Public Health Faculty of Medicine Hacettepe University, 06100 Sihhiye, Ankara, Turkey \\ E-mail: hakana@hacettepe.edu.tr}

\section{Conflicts of interest: none}

Keywords: asylum seeker; challenges; delivery of health care; health care provider; refugee

Received: July 19, 2018

Revised: November 19, 2018

Accepted: November 30, 2018

doi:10.1017/S1049023X19000190

\section{Abstract}

Background: All over the world, migration is affecting millions of people who either choose or are forced to leave their countries of origin. Health is considered to be one of the important aspects of migration that is highly influenced by the circumstances created by mobility within or across countries. In the context of forced migration, it is well-established that refugees are susceptible to various diseases and other health conditions which might occur or deteriorate based on the health systems and health care provision in receiving countries. There is a considerable amount of research on the challenges encountered by refugees in receiving countries, in terms of health care systems and services. However, there seems to be little focus on the health care providers' perspectives of the challenges in health care provision in refugee settings. This review aims to explore some of the articles discussing the challenging issues surrounding refugee health from a provider's perspective.

Methods: A systematic review was conducted through five main online databases: Medline (US National Library of Medicine, National Institutes of Health; Bethesda, Maryland USA); Science Direct (Elsevier; Amsterdam, Netherlands); Scopus (Elsevier; Amsterdam, Netherlands); Sage (Sage Publications; Thousand Oaks, California USA); and Google Scholar (Google Inc; Mountain View, California USA), including only the articles published in English. In addition, grey literature resources available online were used.

Results: Forty-eight articles were included in this review, mainly based on the amount of emphasis they placed on providers' viewpoints in refugee health settings. Most of the articles were retrieved based on their availability in the databases which Hacettepe University (Ankara, Turkey) is subscribed to. Almost all of the articles mentioned the challenges caused by linguistic and cultural barriers, and some focused on providers' limited knowledge and skills, as well as inefficient health care systems. There was little emphasis on ethical discussions, and the physical and emotional impact of caring for refugees on health care professionals. In the few articles discussing ethical and personal dimensions, issues concerning stress, burnout, and safety risks were found to be the recurring themes.

Conclusions: Evidence gathered suggests that the challenges faced by health care providers involve a variety of factors that are specific to health care settings involving refugees, such as linguistic and cultural barriers, and a lack of proper support and training. It seems that the challenges that health care providers face in refugee settings could be further investigated both from professional and personal aspects for a better understanding of refugee health care.

Kavukcu N, Altıntaş KH. The challenges of the health care providers in refugee settings: a systematic review. Prehosp Disaster Med. 2019;34(2):188-196 
economic migrants, and persons moving for other purposes."2 A more specific definition: the definition of refugee, on the other hand, describes a refugee as:

A person who, owing to a well-founded fear of persecution for reasons of race, religion, nationality, membership of a particular social group, or political opinions, is outside the country of his nationality and is unable or, owing to such fear, is unwilling to avail himself of the protection of that country. ${ }^{3}$

Within the overall migration figures, the number of people forced to move from their homes as refugees, asylum seekers, and internally displaced persons reached an unprecedented level with 65.6 million people in $2017 .^{4}$

Migration is a multi-faceted concept which requires attention to vulnerabilities created or exacerbated during all phases of migration: pre-flight, flight, reception, settlement, and resettlement, especially in the context of forced migration..$^{5}$ As in many humanitarian crises, health of the uprooted is at the epicenter of all vulnerabilities, and therefore, should be managed thoroughly at social, cultural, and policy levels.

The models which have been developed to specify the health effects of migration point out three ways in which migration can influence health. The first one explains that migrants' health status becomes compatible with the health status of host communities, showing similarities in health indicators. The second states that the stress migrants have to go through during the settlement of a new environment poses great risk to their health. And finally, the third suggests that the health status of migrants is determined by the interplay of the stressors that motivated or forced people to migrate, and the stressors that are caused by the settlement process in a host country. ${ }^{6}$ Although health problems that migrants have do not differ much from those of the rest of the population in the receiving country, the circumstances in the pre-, while-, and post-migration periods may worsen the health status of individuals. According to the World Health Organization (WHO; Geneva, Switzerland), common health problems experienced during migration are "accidental injuries, hypothermia, burns, gastrointestinal illnesses, cardiovascular diseases, pregnancy and delivery-related complications, diabetes, and hypertension."7 In the European region, among many health risks which migrants are exposed to during the process of migration, the most common are: tuberculosis (TB); Human Immunodeficiency Virus (HIV) infection and viral hepatitis; influenza and other common respiratory infections; vector-borne diseases; water-borne and food-borne diseases; and non-communicable diseases. ${ }^{7}$

In terms of non-communicable diseases, forced migrants have difficulty in accessing health care services and continuous treatment in the form of regular follow-ups and proper medication. Degradation of living conditions and physical injuries also contribute to the occurrence and worsening of non-communicable diseases. $^{7}$

Abuse, sexual assault, and violence are also common in the context of forced migration. Forced migrants are usually subject to sexual abuse, occupational illnesses, psychosocial problems, poverty, and isolation due to illegal acts, such as smuggling and human trafficking, which affects health to a great extent. ${ }^{8}$

In most general terms, the obstacles which refugees encounter can be mainly associated with limited access to health care, both in the country of origin and destination, torture and trauma during migration, and the impact of re-settlement. ${ }^{9}$ A well-founded health response to the uprooted in these health matters urges collective efforts to encourage proper policy making, capacity building, and resource mobilization through a country's own means and that of its partners. For many years, receiving countries, most of which are developing countries, especially in the context of forced migration, have been developing systems to adjust their health care systems to the needs of refugee populations. Many models have been utilized to improve the health status of refugees in different contexts, and these models involve a variety of public health interventions ranging from capacity building through the training of health care providers and employment of medical interpreters to the establishment of refugee-friendly health centers. In all these initiatives, many aspects involving the perspective, view, and attitudes of health care providers working with refugees seem to have been over-looked or subject to little scrutiny. This review, therefore, aims to investigate the challenges of health care providers in the provision of care targeting refugee populations in order to contribute to the current understanding of health care services for refugees.

\section{Methods}

This study mainly involves resources that investigate refugee settings, while there are few other articles focusing on migrant settings that describe contexts posing challenges similar to that of refugees.

Resources were identified from two main strands: a systematic review and purposive research. For systematic review, five main online databases, namely Medline (US National Library of Medicine, National Institutes of Health; Bethesda, Maryland USA); Science Direct (Elsevier; Amsterdam, Netherlands); Scopus (Elsevier; Amsterdam, Netherlands); Sage (Sage Publications; Thousand Oaks, California USA); and Google Scholar (Google Inc.; Mountain View, California USA), were examined. The articles published from January 2000 through April 2018 were taken into consideration, except for one article referred for the definition of the term "vicarious traumatization" used in the review. Only the articles published in English after 2000, and available in the electronic resources of Hacettepe University Library (Ankara, Turkey), were retrieved. For purposive research, articles, publications, and one thesis were identified using Google search engine (Google Inc.; Mountain View, California USA) with the same key words used in the systematic review search, which were: "health care," "professional," "provider," "refugee," "migration," “culture," "barrier," "communication," "training," and "challenges." The search was conducted on titles, abstracts, and keywords. The titles of the records identified through database searching were screened to determine their thematic relevance to health care in the refugee context. Mainly those that made a clear link to this theme were initially retrieved. After duplicates were removed, the screening of the documents involved the thematic link to providers' perceptions and practices.

Abstracts of the selected articles were scanned by two reviewers. The eligibility of the articles was discussed further by two reviewers with the following exclusion criteria: (1) limited information on refugee context/more focus on migration settings; and (2) heavy emphasis on refugee perceptions, barriers to access to health care, and refugee health conditions. The articles with relevant and sufficient information were analyzed in detail, and were annotated by the reviewers for main ideas and important details. An overview of the selection process is given in Figure 1.

In total, 169 articles were retrieved from both research databases and the online search engine (38 from Medline, 39 from ScienceDirect, 52 from Scopus, four from Sage, 26 from Google Scholar, and 10 from Google). After eliminating 28 duplicated articles, 141 articles were screened by two reviewers using abstracts. 


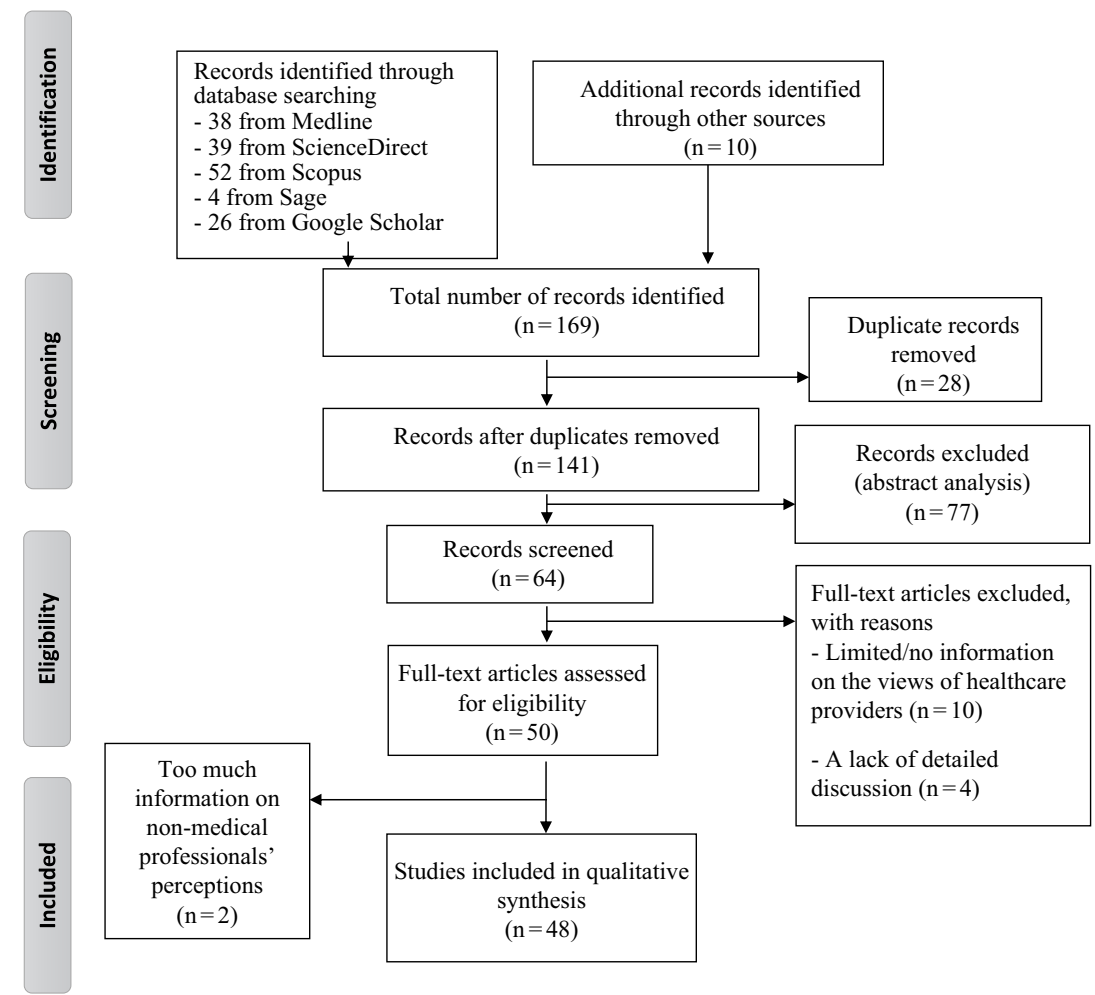

Figure 1. Article Selection Process.

Kavukcu (C 2019 Prehospital and Disaster Medicine Source: Moher D, Liberati A, Tetzlaff J, Altman DG; The PRISMA Group. Preferred Reporting Items for Systematic Reviews and Meta-Analyses: The PRISMA Statement. PLoS Med. 2009;6(7):e1000097.

Of all the articles that were screened, 77 were excluded as they provided limited information on refugee contexts, and they focused only on access to health care from the refugee point-of-view. Then, the remaining 64 records were screened and 14 articles were excluded due to limited information on the provider viewpoint and a lack of detailed discussion on the challenges and perceptions. Two out of 50 eligible articles were excluded because there was little emphasis on health professionals, but instead medical interpreters.

\section{Challenges of Health Care Provision}

Several articles focus on difficulties, particularly in service provision, when explaining challenges identified with health care delivery for refugees. These challenges can be discussed under the following headings: differences in culture and expectations, language barriers, time constraints, lack of knowledge and skills, and system-related obstacles.

\section{Differences in Culture and Expectations}

Cultural competence is central to proper health care provision in regular settings, not just relating to the refugee context. However, its significance becomes even more obvious and compelling when health care is targeted at refugee populations in which beneficiaries mostly share no cultural background and understanding with service providers. Coupled with linguistic barriers, a lack of cultural competence may lead to frustration on the side of the provider and inability to access health care on the side of the refugees.

Literature reveals many examples of cases in which a lack of cultural competence and humility impedes an appropriate way of communication between providers and patients, resulting in challenges in everyday practice of health care providers. In some settings where refugees come from patriarchal cultures, health care providers report that they are unable to employ a holistic approach to addressing health problems, since any attempt to take detailed medical history could be considered threatening and offensive in these cultures. This causes some undisclosed illnesses, like stress and mental disorders, and cases like sexual violence to remain undiagnosed by health providers. ${ }^{10,11}$ In addition to the difficulties in taking proper medical history, the diagnosis of mental health problems is particularly a challenge because of the stigma about receiving treatment for mental health. ${ }^{12}$

Differential understanding of health care due to cultural backgrounds is also a major challenge encountered by health care providers serving culturally diverse populations in day-to-day practice. In a study conducted in Alaska (USA) with Hmong refugees, it was observed that cross-cultural empathy is a key to proper service provision, and different beliefs of Hmong refugees towards health care should be responded through empathy. To exemplify, one patient was reported not to allow a computerized axial tomography/CAT scan during her pregnancy as in her culture, "spirit" could not be contacted before delivery; another refugee patient felt uncomfortable with the medication prescribed since it was not "natural" and was unlike what his ancestors used. Hmong refugees also felt that a close relationship with the health care provider is vital and diagnosis must involve "touching" rather than just asking questions and listening to a patient. ${ }^{13}$

Refugees' differing expectations regarding health care is also reflected in their prioritization of health and willingness to 
collaborate with providers. For most refugees, prevention is an unfamiliar concept. Many refugees stated that they demand health care only when they really need it under life-threatening circumstances, which can be attributed to their considerable reliance on hospitals and other facilities prioritizing acute diseases before, during, and after migration. Therefore, patient involvement can be a huge challenge for health care providers in the provision of preventive health care and proper follow-ups for refugee populations. ${ }^{12-14}$

A study on Somalian immigrant women and their experiences with American doctors provides a good example of how expectations and different understandings can impact health providers' services to culturally diverse groups. To exemplify, the immediacy of diagnosis and prescription in Somalian health care system defines what health care should or should not be like for Somalian refugees, and this causes resistance towards a more involving and prolonged service delivery model in the United States (US) where a number of diagnostic tools are used before the onset of treatment. Disappointed by not getting immediate results, a Somalian patient complains by stating that:

At home [in Somalia], when I am sick, I go to the doctor, I get a shot and I'm fine. Here they keep telling me, 'Come back,' and they're not doing anything. I'm getting worse! ${ }^{15}$

Similarly, medical practices change from culture to culture, and the roles expected from providers may not match the roles assigned to health providers in the host country. In a context dominated by Western medical practice, which depends on collaboration between patient and provider, refugees feel frustrated by the non-paternalistic approach of health care providers. They regard a health care provider as someone with authority and expect to be told what to do. Thus, they develop a sense of mistrust in a patient-centered system where this never happens. ${ }^{16}$

Different understandings of health care could also stem from how the notions of illness and health are conceptualized in various cultures, which is also associated with the level of health literacy among refugees. And it can be said that refugees usually have lower levels of health literacy and limited knowledge on various aspects of health care. ${ }^{17}$

Health-seeking behavior is determined by culture, as well. ${ }^{18}$ In a study on health care providers serving Southeast Asian American immigrant and refugee parents, it was observed that children with mental health problems are taken to families, friends, and spiritual leaders in their community, instead of health care providers. Also, it was noted that parents have a tendency to use alternative traditional remedies instead of medical prescriptions. ${ }^{12}$ In the same fashion, in another study, physicians reported that refugees perceive depression as "sadness," which does not necessarily require formal treatment. ${ }^{19}$

Mistrust is another issue connected to culture which manifests itself from the moment providers begin taking medical histories. Many refugee patients feel that they are asked too many questions or have too many blood tests during screenings, even worrying that their blood is being sold. ${ }^{14}$ Such an attitude certainly hinders providers' obtaining important clinical information and proceeding with the best possible service for refugees.

Cultural norms associated with gender may also pose a challenge to health care providers as they shape the health-seeking behavior and expectations of refugee patients. In most Islamic cultures, female patients prefer to see female doctors or nurses due to cultural and religious beliefs. A study with Iraqi, Afghan, and Iranian refugees and immigrants in Melbourne, Australia shows that women coming from these countries feel more comfortable with female doctors, and their husbands also express preference for them to see female providers. ${ }^{20}$ In emergency care in Sweden, migrant women are reported to have their husbands speak to health care providers on their behalf and to not want male workers close to them when being undressed. ${ }^{21}$ The Centers for Disease Control and Prevention (CDC; Atlanta, Georgia USA) report on Syrian Health Profile also addresses the same tendency in the form of a set of tips for clinicians by mentioning the preference of Syrian patients for same-gender health care providers and "long hospital gowns for modesty, particularly for female patients." 22

Despite the high number of training programs available to equip health care professionals with a culturally sensitive approach to service provision, they may not fully address the issues and help providers in their practice to offer accessible and appropriate services since culture is a vast phenomenon which cannot be defined in a definite way. This puts the health care providers in a complex position where they must manage to "understand" health issues and empower patients to ask questions, make comments, and express their culturally-bound health-related fears, hopes, and goals. ${ }^{18}$ In this regard, cultural competence is certainly a life-long goal as it takes a lot of time and experience with a particular cultural group. Nevertheless, cultural humility and openness can be keys to the initiation of proper communication with culturally diverse groups. Rapport building and active listening prove to be effective ways to gain trust and ensure the appropriateness of services for refugees. Also, increasing the ethnic diversity of health care providers whenever possible, providing services through health care providers who share the same ethnic background as refugee groups, appears to help ensure cultural competence with less time and in a more efficient way. ${ }^{23,24}$

\section{Language Barrier}

The most apparent and widely discussed challenge in serving refugee populations is perhaps related to communication problems due to language barriers. There is considerable amount of research showing the benefits and drawbacks of relying on relatives or utilizing interpreting services. Using relatives for interpreting is found to "improve patient comfort and facilitate communication," whereas it may also jeopardize the "accuracy of history taking and overall patient-provider interaction." ${ }^{14}$ This view is also supported by the argument that having family members translate for refugee patients does not comply with culturally competent care. ${ }^{25}$

The importance of a professional interpreter, even when patients understand the language of the receiving country, was considered by frontline health care providers to be necessary in order for them to catch the nuances in the description of symptoms and support patients who have limited language proficiency. ${ }^{23}$ Medical residents serving Burmese refugees in Indianapolis (Indiana USA) mentioned the significance of easy availability of professional interpreting services through phone, video, or in-person by commenting further on good use of these services with the help of techniques for how to use body language and establish eye-contact with the patient, not the interpreter, during communication. ${ }^{26}$ The challenges surrounding interpretation services vary according to the modality of interpretation. In-person interpretation is found to facilitate a detailed conversation with the involvement of non-verbal cues and the possibility to support the completion of paperwork before examinations. ${ }^{18}$ However, ensuring the quality of interpreting services through the employment of trained professionals is certainly a key to avoiding extra burden on health care providers. ${ }^{27}$ 
An example of poor-quality can be observed in situations where interpreters add their own point-of-view during examinations. As an alternative or complementary to in-person interpreting, phone interpretation could be quite effective due to instantaneous service provision for 24-hours and the protection of women's preservation of anonymity. ${ }^{17,28} \mathrm{On}$ the other hand, interpretation services through phone are usually found to be "too impersonal" and carry a risk of cut-outs and other technological failures. ${ }^{17}$ An interesting aspect of interpreting services for refugees is that interpreters mostly find themselves acting in the roles beyond their medical interpreting functions. This is usually because of the compelling needs that many refugees have, which requires that interpreter relationships be based on longer-term care models. ${ }^{29}$

As is the case with many other aspects of health care provision, interpretation services are not free from the influence of cultural norms. To elaborate, gender roles should be taken into consideration in translation services as well. In maternity care services in Norway, for example, midwives and public health nurses do not prefer to work with male interpreters since they feel that female interpreters can relate to their patients more easily. One nurse working in maternity care service expressed this preference by stating:

It is completely wrong to use men; they don't have the words we use in their vocabulary. I once had a male interpreter and it was a disaster. ${ }^{28}$

In addition, notions that are non-existent in refugees' culture can be hard, or even impossible, to explain for health care providers. A medical director serving Somalian refugee women explained that it was a big challenge for him to tell the patient that she was depressed as there was no equivalent of the word "depression" in their language. ${ }^{23}$

Despite the substantial benefits which professional interpreting services in health care provision offer, it is emphasized that it does not guarantee high standards of care. ${ }^{10}$ The reason for this is that usually interpreters only lift the communication burden to a certain extent by overcoming the linguistic barrier between patient and provider. However, they may not be able to meet the needs of refugee populations, which are specific to a certain group and go beyond "speaking the same language." ${ }^{10,30}$ Therefore, it is crucial that interpreters be trained in appropriate ways to serve a certain refugee group in a culturally sensitive manner.

\section{Time Constraints}

Health visits and consultations create an extremely important opportunity for providers to respond to the health care needs of refugees, especially in terms of mental health. Since these vulnerable groups have additional disease burden compared to regular patients, they require specific attention to "past experience of health care, exposure to traumatic experiences, language, and cultural differences" before and during appointments. ${ }^{9}$ Adopting an approach in which some important steps are incorporated into health care delivery necessitates more effort and time allotted to appointments with refugee populations. As recommended by the Victorian Foundation for Survivors of Torture (Brunswick, Victoria, Australia), initial appointments must involve some crucial steps, like the following:

- Arranging an interpreter;

- Familiarizing refugee patients with the appointment system;

- Calling refugee patients to remind her/him of the appointment;

- Avoiding early morning appointments, as sleeping problems are common among most traumatized refugee patients;
- Promoting overall health assessment;

- Having reception staff inform refugee patients about any delayed appointments; and

- Learning about refugee patients, their background, and possible health problems before the next appointment. ${ }^{9}$

With the necessity to make so many arrangements, even for initial appointments, it can easily be stated that health care providers require much more time for proper service delivery to refugees. Adding the increased time with interpreting services during appointments, it is inevitable that health care providers struggle to find sufficient time to meet the specific needs of refugees in a system where their need for more time is not appreciated.

In one study, this problem made patients report that they cannot share the feelings of depression with their doctors as they are usually rushed, and therefore, their emotional issues were not covered in the check-ups. ${ }^{31}$ According to another study by Fang, et al, a patient stated that the consultations did not last long enough for general practitioners to carry out a thorough assessment, taking the cultural situations into consideration properly. ${ }^{32}$

There is plenty of research investigating consequences of not allotting extra time from the perspectives of refugee health seekers as mentioned above; however, working under time pressure certainly poses several challenges to health care providers as well. A study conducted by Jessen reveals a lack of time for providers to "prepare for visits, educate patients, or address mental health issues." 13 Time limitation can get so extreme that providers usually cannot know whether a refugee or non-refugee patient will show up for the next appointment. ${ }^{13}$

Another major contributor to time restriction is the fact that health care providers need to invest time in building trust with refugee populations to be able to cover all their health-related needs. Also, it is important for them to take time to achieve cultural understanding and explain medical concepts and services made available to refugees in a way that is culturally sensitive. ${ }^{27}$ To improve communication during medical visits, providers must make sure that they employ appropriate strategies to inform refugees clearly about various topics of health, although they are certainly time-consuming. They must employ an effective approach to communication through the use of methods like teach-back, to monitor and check the understanding of patients, or prefer open-ended questions during appointments to encourage patients to give feedback. Although time-consuming, the use of open-ended questions during appointments is extremely important because it was observed that refugees tend to answer "yes" when asked questions that could simply be answered "yes" or "no."14

Many health care providers are aware that their day-to-day work does not only require extended time and duration, but also increased occurrences of appointments when patients are refugees. With the increased number of appointments, which are lengthy and complex, the providers have to carry the burden of the "time taken away from other patient groups." ${ }^{17}$ In one study by Pollock, et al, refugee participants reported that they had been rejected by receptionists because of doctors' perception that serving them is "too time-consuming, emotionally overwrought, and exceptionally demanding." ${ }^{24}$ While analyzing comments like this, it is important to highlight that it would be unfair to interpret such reported cases as acts against code of conduct unless the underlying causes are explored and health care providers' perceptions and attitudes towards appointments with refugees are investigated. 
Refugees' past experiences, culture, and health literacy levels may also have an effect on increased time requirements because of the way they seek health care and utilize health systems. It was reported that time allocation can become an issue with refugees since they tend to miss appointments or arrive late and cause delays in appointment schedules due to their "unfamiliarity with the system, a lack of economic resources, shyness, inability to ask questions, transportation problems, memory issues, anxiety, and mistrust of health care providers." ${ }^{1,33,34}$ A health promotion officer in Australia expresses how these challenges can translate into obstacles in everyday practice in health centers by explaining:

... if a woman misses her appointments a couple of times, or comes extremely late for an appointment, sometimes that can be quite frustrating for receptionists who have to re-book their appointments, yet they don't actually understand the reasons why that might be occurring. ${ }^{35}$

\section{Lack of Knowledge and Skills}

The importance of informing refugees about the health care system and their right to health in the receiving country is reiterated in many sources since the exchange of knowledge in these matters promotes positive encounters with refugees. ${ }^{36}$ Nonetheless, there is relatively less emphasis on the health care providers' knowledge gap in these issues and the cultural dimensions of service provision to refugees as vulnerable groups. Furthermore, support through training and guidance for providers is usually neglected, as shown in many articles discussing the need for more training for health care providers who are "ill-equipped to deal with difficulties in service provision." 27

The following areas can be found in different sources as requiring training and/or support: ${ }^{14,16,17,37-39}$

- Legal processes and entitlements underlying refugee status;

- Socio-political issues of refugees;

- Health systems for refugees;

- Awareness of available resources;

- Cultural sensitivity and competency;

- Understanding of ethnicity and culture and their impact on health care;

- Communicative competence;

- Working properly with interpreters;

- Social inclusion;

- Empathy and gender preferences;

- Trust and rapport building;

- Clinical knowledge and skills in diagnosis, referral, and management of specific health issues of refugees; and

- Mental health care.

A lack of knowledge and skills in the areas mentioned above challenges health care providers in everyday practice. A report by Medact Manchester (London, UK) demonstrates that there is a gap in the knowledge of doctors, nurses, and non-clinical staff working for refugees and asylum seekers in the North-West of England. According to the report, only 21\% of the 198 National Health System (NHS; London, UK) workers who participated in the study could confidently define the terms: "asylum seeker," "failed asylum seeker," "economic migrant," and "refugee." Only around $25 \%$ could explain which groups are entitled to health care services free of charge, and $32 \%$ were unaware that failed asylum seekers were eligible for free emergency care. The majority expressed a lack of confidence in taking histories of trauma and torture, and requested training on different aspects of refugee health and issues surrounding asylum seekers and refugees. ${ }^{40}$ Another study on resident physicians' perceptions shows that most of the residents are fond of serving refugee and migrants; however, they feel concerned about the quality of services. Besides, over one-half of the residents feel they are not knowledgeable enough about immigrant and refugee health. ${ }^{41}$

Regarding the health coverage of refugees, providers can be totally unaware of the refugee entitlement of care in the reception country, or they may be put in a difficult position where they have to decide whether to provide care or not. ${ }^{42} \mathrm{~A}$ study conducted in Canada to examine the health care providers' knowledge of health care coverage for refugees revealed that the overall level of awareness of refugees' health care coverage is quite low among providers, with around two percent of the study population answering all the questions regarding entitlements correctly. ${ }^{43}$ Adding the frequently changing nature of health and legal systems concerning ethnically diverse populations in receiving countries, being and staying knowledgeable as health care providers requires a significant amount of time and effort.

Again in Canada, legal limitations on health care provision to un-insured refugees are reported to raise concerns among most of the providers. ${ }^{44}$ Caught in dilemma between the legislations and the scarcity of resources at one extreme, and the right to health care at the other, providers are confronted with ethical and practical considerations surrounding their practice every day. In contexts where universal access to health care is acknowledged and ensured through legislation, health care provision is smoother and providers are under less pressure. On the other hand, in receiving countries where legislations do not allow free access to health care for undocumented individuals, health care providers still provide services based on humanitarian motives and moral obligations, overlooking legal obligations and risking their own careers. ${ }^{10}$

The complexity of refugee status and the extent of vulnerabilities also create barriers for providers in health care provision when they are not made aware of these with the help of proper training programs. To exemplify, in the cases of sexual assault, coupled with patients' reluctance to share stories of violence due to traumatic and cultural experiences, health care providers are unable to execute proper follow-ups. A study on the health care providers for SubSaharan migrants in Morocco demonstrates that after confrontation with a victim, health care providers do not carry out long-term follow-up due to reasons such as a "lack of time, difficulties in the country, and a lack of control." 11

With limited time to prepare for medical encounters with culturally diverse groups and inadequate training opportunities, health care providers usually lack enough knowledge of refugee culture and good communication skills. As a consequence, they worry that in their encounters, they may be misunderstood and offend refugee patients in certain ways that are unknown to them. ${ }^{33}$ This can even go as far as fearing accusations of racism due to miscommunication and a lack of cultural understanding.

\section{System-Related Obstacles}

Of all the issues discussed under the challenges that health care providers face in health service delivery to refugees, the most influential one is perhaps the health systems relating to refugee health care. This is because it is the health systems that determine the ways in which internal and external factors such as language barrier, a lack of knowledge, or cultural incompetency can become strong enough to interfere with proper service provision. When the systems fall short of responding to the uniqueness of refugee needs, 
this is reflected in providers' avoidance of these cases, and eventually leads to poor health outcomes. ${ }^{34}$

One of the obstacles complicating health care providers' practice is the limited financial resources for refugee health support programs, which hinders any attempts to improve care. Another obstacle is related to limited flexibility, despite the heterogeneity of refugee groups. Unfortunately, it is impossible for health care providers to deliver the complex care required by refugee circumstances in rigid systems, and if the system does not allow for enough flexibility for innovative approaches and more time, providers have to bear the extra emotional and professional burden that the system creates. Finally, the difficulty of refugees in navigating the health system results in "compromised care" and "increased costs," which affect both health seekers and providers in an unfavorable way. ${ }^{45}$

\section{Challenges Threatening Health Care Providers' Well-Being}

There is no doubt that the challenges discussed so far (and beyond the scope of this paper) render health care providers susceptible to burnout and safety risks. It is argued in many studies that health care providers find it emotionally difficult to serve refugee patients and hear their trauma experiences. Providers also feel a sense of helplessness due to their perceived lack of knowledge, skills, and competency in responding to refugee sensitive health care needs, and inability to make a difference in the lives of refugees. ${ }^{17,46}$

A study conducted in the Midlands-based refugee center in the UK demonstrates that working with refugees and asylum seekers increases workload and causes stress as a consequence of time pressure and concerns regarding compromised services. The providers feel that refugee patients can be too demanding and expect too much from the staff, as the system does not allow them to be empowered and increases their dependency on providers. ${ }^{46}$

In addition, moral and legal dilemmas contribute to provider distress to a great extent. Since health care providers are among few people who come in contact with refugee populations, especially at the transition or resettlement periods of movement, they may encounter individuals who have committed unlawful acts or who lack official documents. Having to use own judgement and make legal and moral calculations puts health care providers under considerable pressure and leads to frustration and emotional distress. ${ }^{46}$

The emotional burden of caring for refugees can be put into perspective through a list of common descriptions of emotions that health care providers reported to express the impact that working with refugees has on them. These are the feelings of: "frustration, anger, annoyance, sadness, depression, and feeling down, flat, helpless, and demoralized." ${ }^{46}$

Similarly, health care providers working with trauma survivors are also reported to experience so called "vicarious traumatization," which explains "the signs and symptoms of traumatization similar to those of the victim." 47

Safety may also become an issue of concern for health care providers working with culturally diverse populations. Though not pertaining only to refugee health care settings, the International Committee of the Red Cross (ICRC; Geneva, Switzerland) report provides data showing high incidences of violence against health care providers in the situations of armed conflict and other emergencies between 2012 and 2014. ${ }^{48}$ Despite limited data on the safety of health care in refugee contexts, it would not be totally irrelevant to assume a similar tendency in reception countries, although it may be on a smaller scale. The staff members at Midlands-based refugee center in the UK reported that patients could behave in a violent and aggressive manner, threatening staff, shouting, and throwing things at them. ${ }^{46}$ Also, in emergency care units serving immigrants in Sweden, staff described situations as threatening when husbands react to the discharge of their wives from hospitals, or when patients are involved in drugs and violence. Uniforms are usually regarded as symbols of power in such encounters and make some refugees think that health care providers are actually police officers. In addition, patients' perceived hierarchy of health care staff leads to tensions between refugee patients and nurses when the patients have a lack of trust in staff members other than doctors. Nurses usually find such an attitude frustrating and irritating. Finally, refugee patients interpret waiting times in emergency care as a form of racism and unfair treatment because of their ethnic background, which may again result in outrage and violence. ${ }^{21}$

Regardless of the pessimism dominating most of the articles, it is also true that providers consider serving refugees "personally gratifying" and feel highly motivated by the professional satisfaction they get, enjoy the learning experience of working with multi-cultural groups, and establish fulfilling relationships with refugees. ${ }^{16,33}$ A provider serving refugees in the United States (US) stated:

It is pretty awe-inspiring to be a part of and become a trusted resource for them, and to be able to provide support and help along the way, it is really nice. It is very rewarding. ${ }^{16}$

Another mentioned the opportunity for developing professional skills by stating that it allowed him to practice global health locally, with a mixture of infectious diseases he had to practice and also interaction with interesting cultural characteristics he got familiarized with. ${ }^{16}$ Guhan and Liebling-Kalifani also emphasize the positive outcomes that health care providers serving torture victims can achieve through positive changes in their personality and personal growth. ${ }^{46}$

\section{Limitations of the Review}

The objective of this study is to contribute to the current understanding of refugee health care through a qualitative synthesis of the resources that are available in the context of reviewers in Hacettepe University in Ankara, Turkey. It does not provide generalizable conclusions nor presents a fully comprehensive background for provider perceptions and challenges in refugee health care due to the limitations in data collection.

Firstly, the study only includes articles written in English due to a lack of capacity to evaluate resources written in other languages. This is problematic since refugee-health care is quite contextspecific. Not integrating reports and articles produced in other languages in different parts of the world results in a limited understanding of the phenomenon and poses a risk of bias in terms of representation of certain kinds of ideas. Also, it must be kept in mind that in the systematic review, only five online databases were searched.

Secondly, the search covered the articles published in a time period from January 2000 through April 2018, after which quite a few articles focusing on provider perspectives were published because of an increasing interest in refugee health-related topics. These new articles were unfortunately not included in this paper, since it had already been submitted for peer evaluation.

Thirdly, in the selection of articles, thematic relevance to the study objective was prioritized over methodological assessments in order to generate enough qualitative data for implementers. Since the topic is not much investigated, the reviewers chose to 
draw information from articles that are mainly based on subjective evaluations of research participants as well.

\section{Conclusion}

This review aims to provide an overview of issues surrounding refugee health care from the perspective of a largely neglected stakeholder in current literature: the health care provider. It explored challenges at two different levels, which are health care provision and providers' well-being. Differences in culture and expectations, language barrier, time constraints, lack of knowledge and skills, and obstacles associated with health systems were commonly identified in many of the sources, and are included in this paper as factors that interfere with proper health care provision to refugee patients.
Regarding the challenges affecting personal lives of health care providers, burnout and safety risks are discussed with the experiences of health care providers serving refugees in different parts of the world.

This paper attempts to re-interpret these challenges, discussed mainly with an emphasis on service receivers, and to make an analysis of how they are and might be reflected from providers' stance. It is apparent that more research focusing mainly on provider experiences and viewpoints is needed in order to improve the provision of health care to refugees, advance health systems pertaining to refugee health, and more job satisfaction for health care providers.

\section{References}

1. United Nations. 244 million international migrants living abroad worldwide, new UN statistics reveal. http://www.un.org/sustainabledevelopment/blog/2016/01/ 244-million international-migrants-living-abroad-worldwide-new-un-statistics-reveal/. Accessed April 2018.

2. International Organization for Migration. Key Migration Terms. https://www.iom. int/keymigration-terms. Accessed April 2018.

3. International Organization of Migration Web site. Convention Relating to the Status of Refugees as modified by the 1967 Protocol, Art. 1A(2). 1951. https://www.iom.int/ keymigration-terms. Accessed April 2018.

4. United Nations High Commissioner for Refugees. Forced displacement worldwide at its highest in decades. http://www.unhcr.org/afr/news/stories/2017/6/59 41561f4/ forceddisplacement-worldwide-its-highest-decades.html/. Accessed April 2018.

5. Taylor G. "Migrants and Refugees." In: Papadopolos I, (ed). Transcultural Health and Social Care: Development of Culturally Competent Practitioners. London, UK: Elsevier; 2008:45-64.

6. Llacer A, Zunzunegui MV, del Amo J, Mazarrasa L, Bolumar F. The contribution of a gender perspective to the understanding of migrants' health. JECH. 2007; 61(Suppl 2):4-10.

7. World Health Organization. Migration and health: key issues. http://www.euro.who. int/en/health-topics/health-determinants/migration-andhealth/migrant-health-inthe-european-region/migration-and-health-key-issues. Accessed April 2018.

8. Gushulak BD, MacPherson DW. Health issues associated with the smuggling and trafficking of migrants. J Immigr Health. 2000;2(2):67-78.

9. Foundation House. Document name: Promoting Refugee Health: A Guide for Doctors, Nurses, and other Health Care Providers Caring for People from Refugee Backgrounds (3rd edition). Melbourne, Victoria: Foundation House; 2002, 2007, 2012.

10. Suphanchaimat R, Kantamaturapoj K, Putthasri W, Prakongsai P. Challenges in the provision of healthcare services for migrants: a systematic review through providers lens. BMC Health Serv Res. 2015;15:390.

11. Van den Ameele S, Keygnaert I, Rachidi A, Roelens K, Temmerman M. The role of the healthcare sector in the prevention of sexual violence against sub-Saharan trans migrants in Morocco: a study of knowledge, attitudes and practices of healthcare workers. BMC Health Serv Res. 2013;13:77.

12. Khuu PB, Lee HY, Zhou AQ, Shin J, Lee RM. Healthcare providers' perspectives on parental health literacy and child health outcomes among Southeast Asian American immigrants and refugees. Child Youth Serv Rev. 2016;67, 220-229.

13. Jessen CM. Refugees and healthcare providers in Anchorage, Alaska: understanding cross-cultural medical encounters. Alaska J Antbropol. 2010;8(2):103-113.

14. Kotovicz F, Getzin A, Vo T. Challenges of refugee health care: perspective of medical interpreters, case managers, and pharmacists. JPCRR. 2018;5(1):28-35.

15. Pavlish CL, Noor S, Brandt J. Somali immigrant women and the American healthcare system: discordant beliefs, divergent expectations, and silent worries. Soc Sci Med. 2010;71(2):353-361.

16. MacDonald AP. The Experience of Healthcare Providers Who Care for the Refugee Population. Graduate College dissertations and Theses. Paper 570. 2016.

17. Robertshaw L, Dhesi S, Jones LL. Challenges and facilitators for health professionals providing primary healthcare for refugees and asylum seekers in high-income countries: a systematic review and thematic synthesis of qualitative research. BMJ Open. 2017;7(8):e015981.

18. Rowe J, Paterson J. Culturally competent communication with refugees. Home Health Care Manag Pract. 2010;22(5):334-338.

19. Furler J, Kokanovic R, Dowrick C, et al. Managing depression among ethnic communities: a qualitative study. Ann Fam Med. 2010;8(3):231-236.

20. Neale A, Abu-Duhou J, Black J, Biggs B. Health services: knowledge, use and satisfaction of Afghan, Iranian and Iraqi settlers in Australia. Diversity Health Soc Care. 2007;4:267-276.
21. Hultsjö S, Hjelm K. Immigrants in emergency care: Swedish healthcare staff's experiences. Int Nurs Rev. 2005;52(4):276-285.

22. US Department of Health and Human Services; Centers for Disease Control and Prevention; National Center for Emerging and Zoonotic Infectious Diseases. Syrian Refugee Health Profile. https://www.cdc.gov/immigrantrefugeehealth/pdf/ syrian-health552profile.pdf.2016. Accessed April 2018.

23. Matteliano MA, Street D. Provider's perspectives on cultural competence in ethnically diverse primary care practices. J Fam Med Dis Prev. 2015;1:013.

24. Pollock G, Newbold B, Lafreniere G, Edge S. Discrimination in the doctor's office: immigrant and refugee experiences. Crit Soc Work. 2012;13(2):60-79.

25. Papadopoulos I. "The Papadopoulos, Tilki, and Taylor Model for Developing Cultural Competence." In: Papadopoulos I, (ed). Transcultural Health and Social Care: Development of Culturally Competent Practitioners. London, UK: Elsevier; 2008;7-24.

26. McHenry MS, Nutakki K, Swigonski NL. Effectiveness of cross-cultural education for medical residents caring Burmese refugees. Educ Health. 2016;29(3):50-54.

27. Durston J, Paajanen A. Nursing of refugee and asylum seeker families: an integrated review. Thesis paper. Helsinki, Finland: Metropolia University of Applied Sciences. 2016.

28. Lyberg A, Viken B, Haruna M, Severinsson E. Diversity and challenges in the management of maternity care for migrant women. J Nurs Manag. 2012;20(2):287-295.

29. Drennan V, Joseph J. Health visiting and refugee families: issues in professional practice. J Adv Nurs. 2005;49(2):155-163.

30. Green M. Language barriers and health of Syrian refugees in Germany. Am J Public Health. 2017;107(4):486.

31. Ahmed A, Steward DE, Teng L, Wahoush O, Gagnon AJ. Experiences of immigrant new mothers with symptoms of depression. Arch Womens Ment Health. 2008;11(4): 295-303.

32. Fang ML, Sixsmith J, Lawthom R, Mountain I, Shahrin A. Experiencing 'pathologized presence and normalized absence; understanding health related experiences and access to health care among Iraqi and Somali asylum seekers, refugees and persons without legal status. BMC Public Health. 2015;15:923.

33. Wahoush O. Primary health care: provider perspectives on their services for preschool children in refugee \& refugee claimant families. Prim Health Care. 2013;140(3):1-6.

34. McKeary N, Newbold B. Barriers to care: the challenges for Canadian refugees and their health care providers. J Refug Stud. 2010;23(4):523-545.

35. Mengesha ZB, Perz J, Dune T, Ussher J. Preparedness of health care professionals for delivering sexual and reproductive health care to refugee and migrant women: a mixed methods study. Int J Environ Res Public Health. 2018;174(15):1-13.

36. Mangrio E, Forss KS. Refugees' experience of healthcare in the host country: a scoping review. BMC Health Serv Res. 2017;814(17):1-16.

37. Asgary R, Smith LC, Sckell B, Paccione G. Teaching immigrant and refugee health to residents: domestic global health. Teach Learn Med. 2013;25(3):258-265.

38. Griswold KS. Refugee health and medical student training. Fam Med. 2003;35(9): 649-654.

39. Wray J, Walker L, Fell B. Student nurses' attitudes to vulnerable groups: a study examining the impact of a social inclusion module. Nurse Educ Today. 2008;28(4): 513-520.

40. Lawton K, Lee A, Mahmood N, et al. Healthcare professionals' views and experiences of dealing with refugees and asylum seekers: a survey of North-West practitioners. https://medactmanchester.files.wordpress.com/2017/10/medact_report_2017.pdf. Accessed April 2018.

41. Alpern JD, Davey CS, Song J. Perceived barriers to success for resident physicians interested in immigrant and refugee health. BMC Med Educ. 2016;178(16):1-6.

42. Nies MA, Lim AYW, Fanning K, Tavanier S. Importance of interprofessional healthcare for vulnerable refugee populations. J Immigr Minor Health. 2016;18(5): 941-943. 
43. Ruiz-Casares M, Cleveland J, Oulhote Y, Dunckley-Hickin C, Rousseau C. Knowledge of 603 healthcare coverage for refugee claimants: results from a survey of health service providers in 604 Montreal. PloS ONE. 2016;11(1):1-11.

44. Vanthuyne K, Meloni F, Ruiz-Casares M, et al. Health workers' perceptions of access to care for children and pregnant women with precarious immigration status: health as a right or privilege? Soc Sci Med. 2013;93:78-85.

45. Farley R, Askew D, Kay M. Caring for refugees in general practice: perspectives from coalface. Aust J Prim Health. 2014;20(1):85-91.
46. Guhan R, Liebling-Kalifani H. The experiences of staff working with refugees and asylum seekers in the United Kingdom: a grounded theory exploration. J Immigr Refug Stud. 2011:9(3):205-228.

47. McCann IL, Pearlman LA. Vicarious traumatization: a framework for under standing the psychological effects of working with victims. J Trauma Stress. 1990; 3(1):131-149.

48. ICRC. Violent Incidents Affecting Health Care. Health Care in Danger. January 2012- December 2014. Geneva, Switzerland: ICRC; 2015. https://www.icrc.org/ fre/assets/files/publications/icrc-002-4237.pdf. Accessed April 2018. 